\title{
Efficacy of a Subcutaneous Implantable Cardioverter Defibrillator in a Child with Early Repolarization Syndrome
}

\author{
Moe Taki, ${ }^{1}$ MD, Shota Muraji, ${ }^{1}$ MD, Hitoshi Mori,${ }^{1}$ MD, Taisuke Nabeshima, ${ }^{1}$ MD, Koich Toda, ${ }^{1}$ MD, \\ Yoichi Iwamoto, ${ }^{2} \mathrm{MD}$, Satoshi Masutani, ${ }^{2} \mathrm{MD}$, Takuro Kojima, ${ }^{1} \mathrm{MD}$, Shigeki Yoshiba, ${ }^{1} \mathrm{MD}$, \\ Toshiki Kobayashi, ${ }^{1}$ MD and Naokata Sumitomo, ${ }^{1}$ MD
}

\begin{abstract}
Summary
An early repolarization (ER) pattern or $\mathrm{J}$ waves are considered to be a benign finding observed in the healthy population, however, it has been pointed out that the ER pattern seen in the inferolateral leads could be an independent risk factor for fatal arrhythmias. We present a pediatric case in which early repolarization syndrome (ERS) was suspected due to the presence of ER or J waves in the inferior leads, which eventually disappeared after the administration of pilsicainide. During the follow-up period, several fatal ventricular arrhythmias were recorded after implantation of a subcutaneous implantable cardiac defibrillator (S-ICD). This report describes the efficacy of S-ICDs in a child with an ER pattern after aborted sudden cardiac death.
\end{abstract}

(Int Heart J 2021; 62: 919-923)

Key words: J wave syndrome, Ventricular fibrillation, Pilsicainide, Child

A lthough an early repolarization (ER) pattern or $\mathrm{J}$ wave syndrome may contribute to sudden cardiac death, ${ }^{1-3)}$ it is considered to be a benign finding, present in 1 to $8 \%$ of the general population, and mainly observed in young athletes, adolescents, and the black race. ${ }^{4-7)}$ We present a pediatric case in which early repolarization syndrome (ERS) was suspected due to the presence of ER in the inferior leads, which eventually disappeared after the administration of pilsicainide. During the follow-up period, several fatal ventricular arrhythmias were recorded after implantation of a subcutaneous implantable cardiac defibrillator (S-ICD). A pilsicainide administration test may differentiate malignant ERS from a benign ER pattern.

\section{Case Report}

A 12-year-old boy suddenly fell down while standing after running $100 \mathrm{~m} 4$ times at school, and he immediately underwent cardiopulmonary resuscitation (CPR) by a school teacher, and a $155 \mathrm{~J}$ automated electrical defibrillator (AED) shock successfully terminated the ventricular fibrillation (VF). Sinus rhythm was resumed after transient complete atrio-ventricular (AV) block, however, VF was re-initiated after manifestation of the ER, and a second AED shock was delivered (Figure 1). He had resumed spontaneous respiratory circulation when the emergency team had arrived 17 minutes after starting the CPR. His
Glasgow coma scale was E1V1M4, and he was transferred to a pediatric intensive care unit under artificial ventilation by endotracheal intubation. He underwent 3 days of brain hypothermia therapy and was then transferred to our hospital for further evaluation and treatment of the cardiac arrest.

An electrocardiogram (ECG) on admission showed sinus rhythm with a heart rate of $81 \mathrm{bpm}$, QT $0.38 \mathrm{sec}-$ onds, QTc 0.41 seconds, and mild negative $\mathrm{T}$ waves in lead III. Cardiac catheterization excluded any organic heart disease. Cardiac magnetic resonance imaging showed normal cardiac function, without any findings of myocardial injury, myocardial infarction, or myocardial edema. The creatine kinase MB fraction value (1.8 ng/ $\mathrm{mL}$ ) was also within normal range. None of the ventricular arrhythmias were induced by a treadmill exercise test, and a Holter electrocardiogram showed only 5 beats of supraventricular premature contractions per day. The signal-averaged electrocardiogram showed all the values were within normal range (root mean square voltage of the last $40 \mathrm{msec}$ was $69.5 \mu \mathrm{V}[>15.0 \mu \mathrm{V}]$, low amplitude signals below $40 \mu \mathrm{V}$ were $21 \mathrm{msec}[<39 \mathrm{msec}]$, and the filtered QRS duration was $90 \mathrm{msec}[<105 \mathrm{msec}])$. No ventricular tachycardia (VT) or VF was induced by burst stimulation or up to triple extra-stimulation from the right ventricular apex either in the control state or under a continuous infusion of isoproterenol, or epinephrine. He had no family history of unexpected syncope or sudden car-

From the ${ }^{1}$ Department of Pediatric Cardiology, Saitama Medical University International Medical Center, Saitama, Japan and ${ }^{2}$ Department of Pediatrics, Saitama Medical University, Saitama, Japan.

Address for correspondence: Naokata Sumitomo, MD, Department of Pediatric Cardiology, Saitama Medical University International Medical Center, 1397-

1 Yamane Hidaka, Saitama 350-1298, Japan. E-mail: sumitomo@saitama-med.ac.jp

Received for publication September 29, 2020. Revised and accepted February 3, 2021.

Released in advance online on J-STAGE July 17, 2021.

doi: 10.1536/ihj.20-661

All rights reserved by the International Heart Journal Association. 


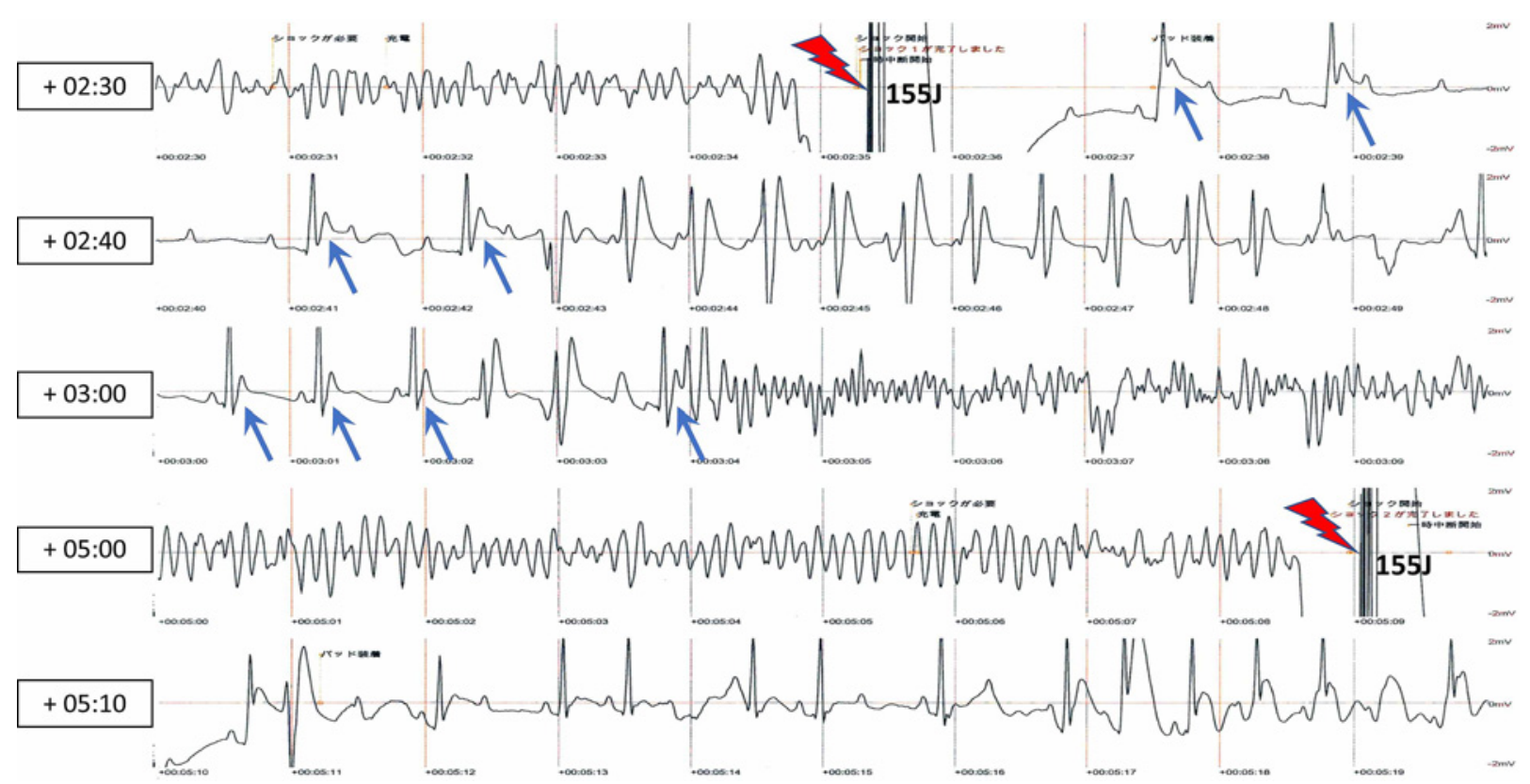

Figure 1. Monitor recording from the automated electrical defibrillator. VF was successfully terminated by a $155 \mathrm{~J}$ direct current (DC) shock (red thunder bolt arrows). During complete AV block with a junctional rhythm and several beats of sinus rhythm, the recording showed marked J waves (blue arrows), then it suddenly degenerated into VF, and a second DC shock was delivered (thunder bolt arrow).

diac death. Genetic testing for $K C N Q 1, K C N H 2$, SCN5A, and $R y R 2$ showed no genetic abnormalities. Interestingly, the ER that were observed in leads II, III, and aVF disappeared, and become small s waves after loading $50 \mathrm{mg}$ of pilsicainide, which is a pure $\mathrm{Na}$ channel blocker. An increase in the amplitude of the late R' at 1 intercostal space above the right precordial leads was observed simultaneously after the administration of pilsicainide, but no coved type ST-T change presented (Figure 2). From that testing, long QT syndrome (LQTS), Brugada syndrome (BRS), catecholaminergic polymorphic ventricular tachycardia (CPVT), and short QT syndrome (SQTS) were excluded, and the patient was diagnosed with idiopathic ventricular fibrillation (IVF) (suspected of ERS).

Implantation of an implantable cardioverterdefibrillator (ICD) was indicated as a class I indication in this patient (height: $161.3 \mathrm{~cm}$, weight: $54.1 \mathrm{~kg}$ ), and he was implanted with an S-ICD (Boston Scientific, EMBLEM $^{\mathrm{TM}}$ MRI S-ICD, MA, USA). One year after implantation of the S-ICD, he felt light headedness at the end of light exercise, and 34 beats of VT at a rate of $300 \mathrm{bpm}$ were recorded by the remote monitor system of the $\mathrm{S}$ ICD, but a shock was not delivered because the VT spontaneously terminated in 6.5 seconds. However, 20 months after implantation of the S-ICD, 70 beats of a nonsustained VT were detected by S-ICD, and the shock was delivered, but the VT spontaneously terminated just before the delivery of the shock (Figure 3A). At that time, a treadmill exercise test provoked ventricular salvos with right bundle branch block and a superior axis morphology (Figure 3B). Interestingly, there was no increase in the amplitude of the ER before the initiation of VT. After this event, $150 \mathrm{mg}$ of flecainide followed by $60 \mathrm{mg}$ of nadolol were prescribed to prevent the occurrence of VT and to decrease the chance of any appropriate shocks from the SICD.

\section{Discussion}

Brugada syndrome (BRS) is the most common disease that may cause VF in young adults. ${ }^{89}$ ) However, in this patient, ECGs at rest and after using pilsicainide exhibited no coved type ST-T changes, and there was no genetic anomaly of the SCN5A gene, which ruled out the possibility of BRS. LQTS and SQTS are other possible mechanisms of VF in young people, ${ }^{9)}$ however, there was no QT prolongation or shortening in the resting ECG or in the ECG during exercise, which may have excluded the possibility of LQTS and SQTS. CPVT is a well known arrhythmia, which may cause sudden cardiac death in young patients, ${ }^{9-11)}$ however, monomorphic ventricular premature beats or salvos were induced during the follow-up exercise test in this patient, monomorphic VT was also recorded by the S-ICD remote monitor system, no ventricular arrhythmias were induced during isoproterenol or epinephrine infusion, and genetic testing for $R y R 2$ revealed no genetic anomalies in this patient, and therefore, we concluded the possibility of CPVT was unlikely.

The presence of ER in the resting ECG and magnification of the amplitude of the ER before the initiation of $\mathrm{VF}$ in this patient suggested the possibility of ERS. Although ER is determined by the presence of $\mathrm{J}$ waves with $\geq 0.1 \mathrm{mV}$ notches or slurs of the terminal portion of the QRS complex in at least two adjacent leads, ${ }^{2,9)}$ this patient had ER in only the inferior leads. Kawata, et al. reported that pilsicainide administration showed effects in patients with BRS associated with ERP and patients with ERS. ${ }^{12}$ The amplitude of the ER was attenuated in 13 of 14 pa- 
A

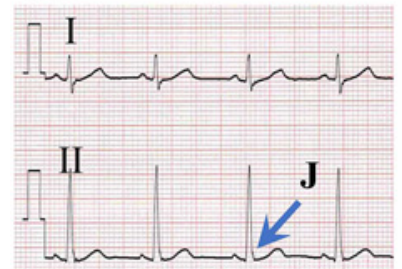

III

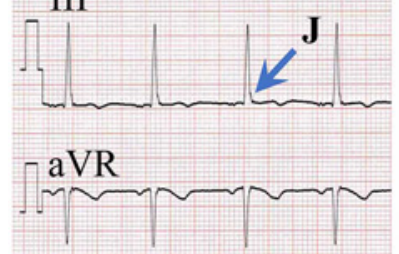

$-\mathrm{aVL}$
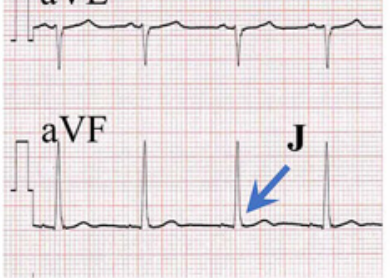

B

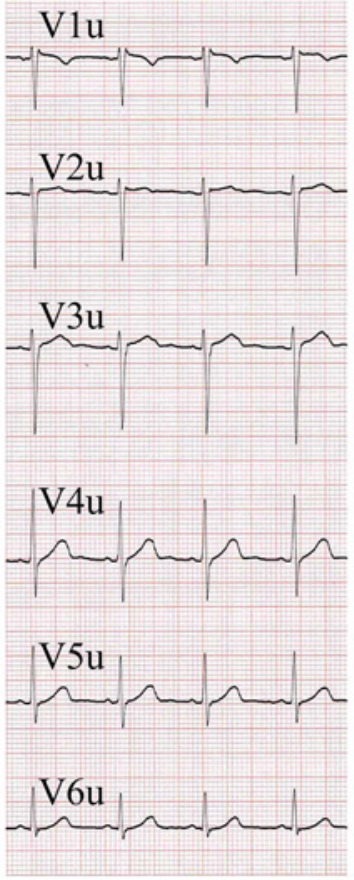

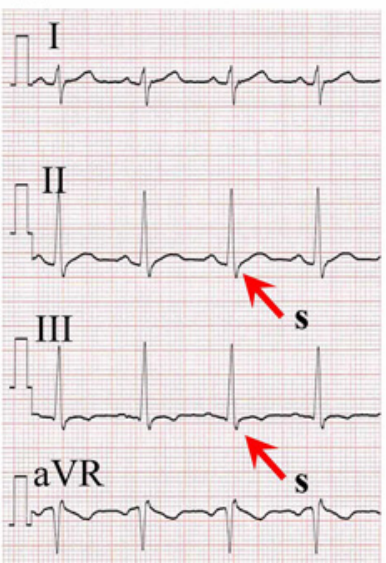
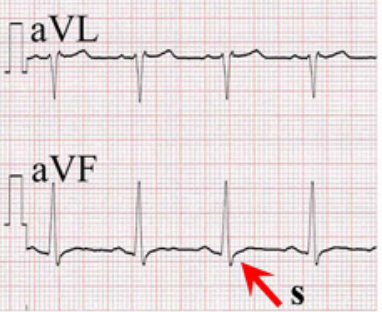
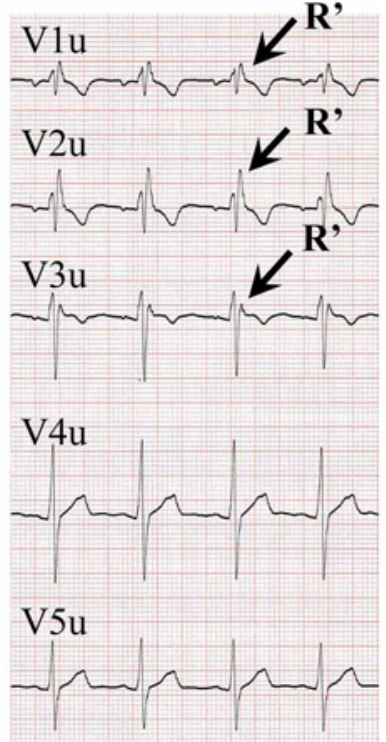

V6u

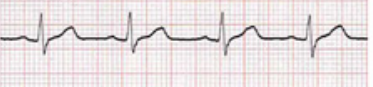

Figure 2. Electrocardiograms before and after pilsicainide. The small $\mathrm{J}$ waves (blue arrows) in leads II, III, and aVF existed in the control state, and there were no coved type ST-T changes in the precordial leads placed 1 intercostal space higher (V1u to V6u). After administration of $50 \mathrm{mg}$ of pilsicainide, the J waves disappeared and changed into small s waves (red arrows). An increase in the amplitude of the late R' in V1u to V3u was also observed (black arrows). V1u to V6u $=1$ intercostal space above the V1 to V6.

A

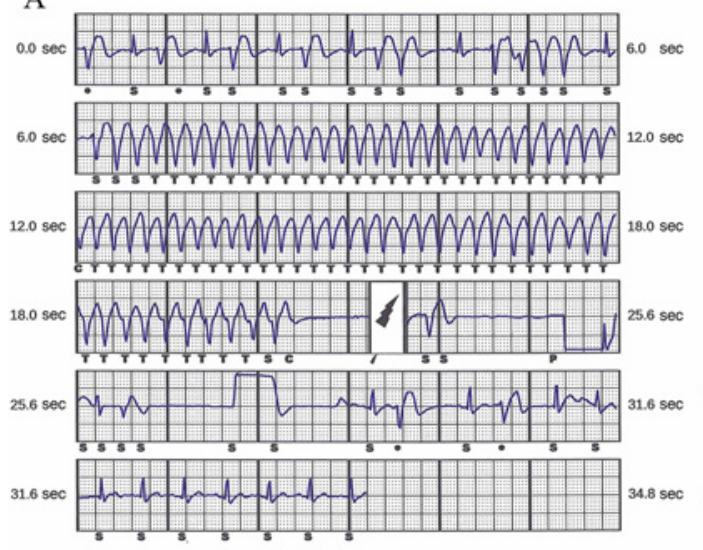

B

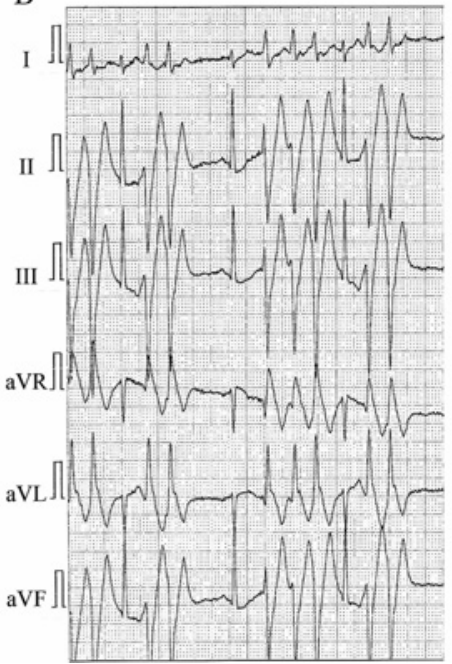

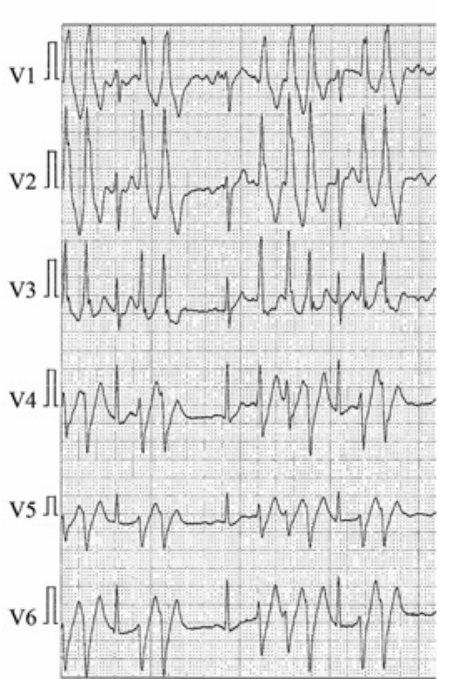

Figure 3. Remote monitoring of the subcutaneous implantable cardiac defibrillator and exercise induced ventricular tachycardia. A: Twenty months after implantation of the S-ICD, he suddenly felt a shock while going up the stairs at school. The remote monitoring showed bigeminal premature ventricular contractions (PVCs), PVC couplets, PVC triplets, and PVC salvos, and then 70 beats of a ventricular tachycardia (VT) at a rate of $300 \mathrm{bpm}$ were suddenly induced. The VT terminated spontaneously within 20 seconds, however, an S-ICD shock was delivered (thunder bolt arrow). B: A right bundle branch block, superior axis morphology nonsustained VT was induced during a treadmill exercise test.

tients $(93 \%)$ with ERS and in 5 of 5 patients $(100 \%)$ with BRS. In some patients, along with the disappearance of the ER, s waves appear in the same leads as in ERS patients. They postulated that this attenuation or disappear- ance of the ER was largely due to the transmural conduction delay caused by $\mathrm{Na}$ channel blockers. Our patient also had ER in the inferior leads, which disappeared and s waves appeared after a pilsicainide injection. Kamakura, 
et al. reported that 38 patients with inferolateral ER and prior VF did not have any recorded coved type ST-T changes. ER is attenuated in patients in which saddleback type ST-T changes are only detected in high intercostal precordial leads and patients without saddleback type ST$\mathrm{T}$ changes in any lead at baseline or after a pilsicainide injection. They concluded that ER with a BRS ECG may have the risk of arrhythmic events. ${ }^{13)}$ In other words, ERS and BRS may be differentiated from each other by the finding of attenuation of the ER after using pilsicainide.

IVF is a class I indication for an ICD implantation. Dalos, et al. reported that in patients with ER who had a VF event and an ICD implantation, 50\% of the patients underwent appropriate defibrillation therapy over an average follow-up of $11.2 \pm 6.7$ years. ${ }^{14)}$ Therefore, the necessity of an ICD implantation in those patients has been suggested. Ahmed, et al. reported that ER is acknowledged in $41 \%$ of healthy adolescent group patients aged 13-18 years, which is higher than the prevalence rate in adults. ${ }^{7)}$ They also stated that there was no statistically significant difference in the presence or absence of ER with respect to the age, race, sex, height, weight, systolic blood pressure, diastolic blood pressure, or history of syncope. In addition, he concluded that there was no association with the echocardiographic findings or ER findings in those with a family history of fatal cardiac events. Hasegawa, et al. reported that bradycardia and hypothermia may increase the voltage of the $\mathrm{J}$ waves in young adult patients surviving aborted sudden cardiac death. ${ }^{15)}$ Kimura, et al. also reported that R-R interval prolongation and being male were independent factors associated with the presence of J waves. ${ }^{16)}$

We implanted an S-ICD in this patient. The S-ICD is a relatively new device, and reports of S-ICDs in children are limited. Silvetti, et al. reported a follow-up evaluation of 15 patients (age 14 to 28 years) with S-ICD implantations, and a higher complication rate was noted in the 3 incision group than the 2 incision group. Also, patients with a body mass index $(\mathrm{BMI})<20$ tended to have more complications than those with a BMI of $\geq 20 .{ }^{17)}$ Although the device size is bigger than a traditional ICD, an S-ICD is preferable in young patients with IVF when the patients do not need ventricular pacing, because it may avoid serious intravenous lead troubles, such as sepsis, cardiac perforations, and venous occlusions. We implanted an S-ICD in this patient with the 2 incision method, and his BMI was 20.3. There have been no complications during 2 years of follow-up, VT was first detected by the remote monitoring system of the S-ICD, and an appropriate shock was delivered during the follow-up period. Before the initiation of this VT, there was no increase in the amplitude of the ER that may have indicated another mechanism during the occurrence of this VT, such as some myocardial damage during the resuscitation of this patient.

ER is found in about 1 to $13 \%$ of the normal population, but it is detected in 15 to $70 \%$ of patients with IVF. ${ }^{9)}$ Therefore, ER in the inferior or lateral leads in itself cannot be a risk factor for fatal arrhythmias in children and adolescents. On the other hand, the initial occurrence of $\mathrm{VF}$ at an age $<16$ years could be an independent risk factor for a recurrence of a fatal arrhythmia in patients with
IVF, and those children should be carefully evaluated for the cause of the VF and receive therapeutic management. ${ }^{18)}$ Takayama, et al. also reported a 12 year-old ERS patient with a gain of function KCND3 mutation who experienced a VF event. ${ }^{19)}$ To the best of our knowledge, this is only the second pediatric ERS case report.

\section{Conclusion}

This was an IVF case report of a child without any known genetic mutation. The findings suggested that ER in itself has a minor risk of a fatal cardiac event in children but has the chance of resulting in a fatal arrhythmic event in children. In this patient, we successfully recorded a ventricular arrhythmia using S-ICD remote monitor interrogation. Pilsicainide administration testing may differentiate malignant ERS from a benign ER pattern. The follow-up of the pediatric ER population and observation of the incidence of long-term cardiac events and prognosis are mandatory.

\section{Acknowledgments}

The authors thank their colleagues at Saitama Medical University International Medical Center who performed the implantation of the S-ICD in this patient. We also thank Mr. John Martin for his help in the preparation of the manuscript.

\section{Disclosure}

Conflicts of interest: The authors have no conflicts of interest or funding to disclose.

\section{References}

1. Haissaguerre M, Derval N, Sacher F, et al. Sudden cardiac arrest associated with early repolarization. N Engl J Med 2008; 358: 2016-23.

2. Tikkanen JT, Anttonen O, Junttila MJ, et al. Long-term outcome associated with early repolarization on electrocardiography. $\mathrm{N}$ Engl J Med 2009; 361: 2529-37.

3. Wu SH, Lin XX, Cheng YJ, Qiang CC, Zhang J. Early repolarization pattern and risk for arrhythmia death: a meta-analysis. J Am Coll Cardiol 2013; 61: 645-50.

4. Pelliccia A, Culasso F, Di Paolo FM, et al. Prevalence of abnormal electrocardiograms in a large, unselected population undergoing pre-participation cardiovascular screening. Eur Heart J 2007; 28: 2006-10.

5. O'Neal WT, Wang YG, Wu HT, et al. Electrocardiographic J Wave and Cardiovascular Outcomes in the General Population (from the Atherosclerosis Risk In Communities Study). Am J Cardiol 2016; 118: 811-5.

6. Pérez-Riera AR, Abreu LC, Yanowitz F, et al. "Benign" early repolarization versus malignant early abnormalities: clinicalelectrocardiographic distinction and genetic basis. Cardiol J 2012; 19: 337-46.

7. Ahmed H, Czosek RJ, Spar DS, Knilans TK, Anderson JB. Early Repolarization in Normal Adolescents is Common. Pediatr Cardiol 2017; 38: 864-72.

8. Brugada P, Brugada JJ. Right bundle branch block, persistent ST segment elevation and sudden cardiac death: A distinct clinical and electrocardiographic syndrome: A multicenter report. Am 
Coll Cardiol 1992; 20: 1391-6.

9. Priori SG, Wilde AA, Horie M, et al. HRS/EHRA/APHRS expert consensus statement on the diagnosis and management of patients with inherited primary arrhythmia syndromes: document endorsed by HRS, EHRA, and APHRS in May 2013 and by ACCF, AHA, PACES, and AEPC in June 2013. Heart Rhythm 2013; 10: 1932-63.

10. Leenhardt A, Lucet V, Denjoy I, et al. Catecholaminergic polymorphic ventricular tachycardia in children. A 7-year follow-up of 21 patients. Circulation 1995; 91: 1512-9.

11. Sumitomo N, Harada K, Nagashima M, et al. Catecholaminergic Polymorphic Ventricular Tachycardia in Children and Young Adults: Electrocardiographic characteristics and optimal therapeutic strategies to prevent sudden death. Heart 2003; 89: 6670 .

12. Kawata H, Morita H, Yamada Y, et al. Prognostic significance of early repolarization in inferolateral leads in Brugada patients with documented ventricular fibrillation: A novel risk factor for Brugada syndrome with ventricular fibrillation. Heart Rhythm 2013; 10: 1161-8.

13. Kamakura T, Wada M, Nakajima I, et al. Significance of electrocardiogram recording in high intercostal spaces in patients with early repolarization syndrome. Eur Heart J 2016; 37: 630-
7.

14. Dalos D, Fiedler L, Radojevic J, Sponder M, Dichtl W, Schukro C. Prevalence of early repolarization syndrome and long-term clinical outcome in patients with the diagnosis of idiopathic ventricular fibrillation. Heart Vessels 2019; 34: 625-31.

15. Hasegawa K, Miyazaki S, Morishita T, et al. A slower heart rate and therapeutic hypothermia unmasked early repolarization syndrome in a ventricular fibrillation survivor. Int Heart J 2019; 60: $185-8$.

16. Kimura Y, Yamada M, Hanada K, et al. Relationship between serum eicosapentaenoic acid levels and $\mathrm{J}$-waves in a general population in Japan. Int Heart J 2018; 59: 736-40.

17. Silvetti MS, Pazzano V, Verticelli L, et al. Subcutaneous implantable cardioverter defibrillator: is it ready for use in children and young adults? A single-centre study. Europace 2018; 20: 1966-73.

18. Conte G, Belhassen B, Lambiase P, et al. Out-of-hospital cardiac arrest due to idiopathic ventricular fibrillation in patients with normal electrocardiograms: results from a multicentre long-term registry. Europace 2019; 21: 1670-7.

19. Takayama K, Ohno S, Ding WG, et al. A de novo gain-offunction KCND3 mutation in early repolarization syndrome. Heart Rhythm 2019; 16: 1698-706. 